\title{
FOREWORD
}

\section{Special Section on Multimedia QoS Evaluation and Management Technologies}

With advances in IP and multimedia technologies, various multimedia services such as video streaming, videophones, and videoconferencing services are being developed and provided. Properly evaluating and managing users' perceptions of multimedia quality of service (QoS) to provide comfortable multimedia services is important because their performances are not generally guaranteed in present IP networks. Moreover, in the next generation IP network, commercial multimedia services must assure an acceptable QoS. Properly designing or controlling the multimedia application or network is also important.

The purpose of this special section is to present $R \& D$ activities and innovations that advance the progress of multimedia QoS evaluation and management technologies. We have received 37 paper submissions from 8 countries, about half of which came from outside Japan. After careful review and discussion among editorial committee members, 15 papers have been selected for publication. The selected papers mainly cover users' perceptual QoS assessment, QoS monitoring/measurement, and QoS control technologies relevant to multimedia services such as IP-phone, video streaming, videophone, and on-line games. The section invited two papers that provide perspectives on current R\&D on multimedia QoS evaluation.

I hope that this section will serve as good literature that shows the overall progress on R\&D in multimedia QoS evaluation and management.

Finally, I would like to express my deepest appreciation to all the authors for their contributions. My sincere thanks are also extended to the difficult review work of the reviewers and the editorial committee members.

Editorial Committee Members

Secretary: Takanori Hayashi (NTT)

Guest Editors: Yutaka Ishibashi (Nagoya Inst. Tech.), Eisaburo Itakura (SONY), Ryoichi Kawada (KDDI),

Nobuhiko Kitawaki (Tsukuba Univ.), Kenji Sugiyama (Seikei Univ.), Eisuke Nakasu (NHK), Boon

Choong Seng (NTT DoCoMo), Kenichi Hosaka (ATR), Yuukou Horita (Toyama Univ.), Mitsuru

Miyauchi (Chiba Inst. Tech.), Tsutomu Murase (NEC), Katsunori Yamaoka (Tokyo Inst. Tech.), and Toshiyuki Yoshida (Fukui Univ.)

Hisao Yamamoto, Guest Editor-in-Chief

\begin{abstract}
Hisao Yamamoto (Member) received the B.E., M.E., and Dr. E. degrees in Electrical Engineering from Keio University, Tokyo, Japan, in 1974, 1976, and 1984, respectively. Since April 1976, he has been with the Musashino Electrical Communication Laboratory of NTT, where he has been engaged in research mainly on digital phase-locked loop circuits, optical switching systems, network control systems, network operation systems, telecommunicationuser behavior and multimedia network services. From 2002, he has served as a professor at the Musashi Institute of Technology, Dept. of Systems Information Engineering. Dr. Yamamoto is a senior member of the Communication Society of IEEE and a member of the Operations Research Society of Japan.
\end{abstract}

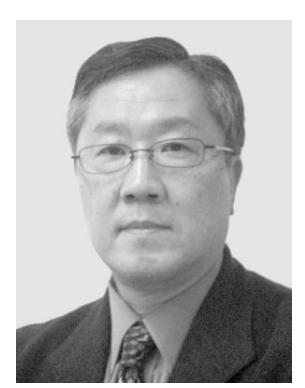

